\title{
Are adults just big kids? Can the newer paediatric weight estimation systems be used in adults?
}

\author{
O Akinola, ${ }^{1,2}$ BSc (Anatomy), MBBS, MMed (Emergency Medicine), FCEM (SA); M Wells, ${ }^{1}$ MB BCh, PhD, FCEM (SA), DipPEC (SA); \\ P Parris, ${ }^{1}$ MB BCh, MMed (Emergency Medicine), FCEM (SA); L N Goldstein, ${ }^{1}$ MB BCh, MD, FCEM (SA), Cert Critical Care (SA) \\ ${ }^{1}$ Division of Emergency Medicine, School of Clinical Medicine, Faculty of Health Sciences, University of the Witwatersrand, Johannesburg, \\ South Africa \\ ${ }^{2}$ Department of Emergency Medicine, University College Hospital, Ibadan, Nigeria
}

Corresponding author: L N Goldstein (drg666@gmail.com)

\begin{abstract}
Background. The weight of a patient is an important variable that impacts on their medical care. Although some drugs are prescribed on a so-called 'adult dose' basis, we know that adults come in all shapes and sizes - a 'one-dose-fits-all' approach is not necessarily appropriate. As a measured weight may not always be available, an alternative method of accurately estimating weight is required.

Objectives. To assess and compare the accuracy of weight estimations in adults by patient self-estimation, the Mercy method, Buckley method, Broca index and PAWPER XL-MAC (paediatric advanced weight prediction in the emergency room eXtra length/eXtra large midarm circumference) method.

Methods. This was a prospective, cross-sectional study conducted at a tertiary academic hospital in a metropolitan area of Johannesburg, South Africa. Anthropometric variables of height, abdominal circumference, thigh circumference, mid-arm circumference and humeral length were measured. These variables were then applied to the various weight estimation methods and compared with the patient's actual weight.

Results. There were 188 adult patients included in the study. None of the methodologies evaluated in this study achieved the recommended $>70 \%$ of weight estimations within $10 \%$ of the patient's actual weight (PW10). The Mercy method was the closest to achieving greater than the recommended $95 \%$ for weight estimation falling within $20 \%$ of the patient's actual weight (PW20). The PW20 for the Mercy method was $91.5 \%$. The PAWPER XL-MAC and patient self-estimate methods achieved a PW20 of $85.1 \%$ and $86.1 \%$, respectively. The Broca and Buckley methods performed poorly overall.

Conclusions. None of the evaluated weight estimation methodologies was accurate enough for use in adult weight estimation. The Mercy and PAWPER XL-MAC methodologies both showed significant promise for use in adult weight estimation, but need further refinement. Although patient self-estimates were similarly accurate to those found in previous studies, they were not an accurate option; self-estimations would remain the first choice if the patient was able to provide such an estimation. The Broca index and Buckley method cannot be recommended owing to their poor performance.
\end{abstract}

S Afr Med J 2021;111(2):166-170. https://doi.org/10.7196/SAMJ.2021.v111i2.15061

The weight of a patient is an important variable that impacts on their medical care. It is the basis for the calculation of many medication dosages, nutritional status, estimation of renal function and intravenous fluid regimens. ${ }^{[1]}$ Use of the correct weight to determine medication dosages is pivotal for life-saving interventions in an emergency, but also for drug dosing for inpatient and outpatient management. ${ }^{[1,2]}$ Although some drugs are prescribed on a so-called 'adult dose' basis, adults come in all shapes and sizes - a 'one-dosefits-all' approach is not always appropriate. Therefore, many drugs for adults should be based on their total body weight, as is the case for children: we should treat adults more like big kids.

Ideally, all patients should be weighed to obtain an accurate weight. However, this is not always practical, especially in an emergency. Acute drug dose calculations therefore commonly rely on selfestimates of a patient's weight, family estimates or alternatively healthcare provider estimates of weight. However, most of these methods have been shown to be inaccurate. ${ }^{[2,3]}$

Few methods have been researched for adult weight estimation, although various formulas, such as the Broca index and the Buckley method, have been suggested for this purpose. ${ }^{[4-6]}$ Numerous studies, however, have been conducted in children to evaluate methods to estimate their weight in the emergency setting. ${ }^{[7]}$ These have resulted in the development of length-based weight estimation devices, such as the PAWPER XL-MAC (paediatric advanced weight prediction in the emergency room eXtra length/eXtra large mid-arm circumference) method and the Mercy method. ${ }^{[8,9]}$ These weight estimation techniques utilise recumbent length or humeral length (as a surrogate for recumbent length) and mid-arm circumference (MAC) (as an indicator of body habitus) to estimate a child's weight. These have shown promise with regard to accuracy and usability in children, but have recently also been evaluated for use in adults. ${ }^{[10]}$

The aim of this study was to assess and compare the accuracy of weight estimations in adults by patient self-estimation, the Mercy method, Buckley method, Broca index and PAWPER XL-MAC method.

\section{Methods}

Study design and setting

This was a prospective, cross-sectional study conducted at a tertiary academic hospital in a metropolitan area of Johannesburg, South Africa (SA).

\section{Study participants}

A non-consecutive convenience sample was used to recruit the study participants. These consisted of adult patients ( $\geq 18$ years of age), who were triaged green according to the SA triage scale, were not 
acutely ill, presented to the hospital general outpatient polyclinic and the emergency department. Patients unable to stand on a scale or who had conditions that would add to their weight, such as pregnancy, oedema or anasarca, or any deformity or disability that would affect measurement of height, weight, humeral length, MAC, abdominal circumference $(\mathrm{AC})$ or thigh circumference (TC), were excluded.

After consent was obtained, participants were asked to give a verbal estimate of their weight. They were then asked to remove heavier articles of clothing, jackets, boots, blankets or loose robes for measurement purposes. The measurements taken were height, with a mechanical telescopic measuring rod (SECA 222, Germany), AC, TC, MAC, humeral length and, lastly, their actual weight, with an electronic flat scale (SECA 803, Germany). All measurements were taken by a single researcher (OA). The flowchart of the recruitment process and procedure is illustrated in Fig. 1.

\section{Weight estimation methods \\ Mercy method}

The Mercy method uses anthropometric surrogates for stature and body habitus, humeral length and MAC to estimate paediatric body weight. ${ }^{[9]}$ The variables are assigned a partial or fractional weight according to a table derived by the developers of the method. The total estimated body weight for the child is then generated by the sum of the two partial weights. ${ }^{[9,11]}$

For example, if a patient had a humeral length of $31 \mathrm{~cm}$ and a MAC of $33 \mathrm{~cm}$, the corresponding partial weights on the Mercy table are $23.7 \mathrm{~kg}$ and $44.8 \mathrm{~kg}$, respectively. The total weight for the patient would then be calculated as $23.7 \mathrm{~kg}+44.8 \mathrm{~kg}=74.5 \mathrm{~kg}{ }^{[9]}$
The table used for the Mercy method has a maximum humeral length of $45 \mathrm{~cm}$ and a MAC of $47 \mathrm{~cm}$, corresponding to partial weights of $53 \mathrm{~kg}$ and $96 \mathrm{~kg}$, respectively. ${ }^{[1]}$ In this study, if the patient's measurements exceeded these parameters, the maximum values were used.

\section{PAWPER XL-MAC method}

The PAWPER XL-MAC method is one of the most accurate methods for estimating paediatric weight in an emergency. ${ }^{[8,12]}$ It is a length-based, habitus-modified weight estimation method. The initial weight estimate is derived by measuring the length of the patient with the tape. The patient's MAC is then measured. The weight assigned to the patient is adjusted based on the habitus modification from the MAC measurement, which is found on the tape. A video demonstrating the measurement technique can be found at: https://www. youtube.com/watch?v=02_L8S0_e7g. As the PAWPER XL-MAC tape was designed to be used in paediatric patients, the length only extends up to $180 \mathrm{~cm}$. If the patient's height exceeded this parameter, the maximum values on the tape were used.

\section{Broca index}

The Broca index (also known as the Broca formula) is an equation that was originally created to determine ideal body weight based on the anthropometric measures of height and weight. ${ }^{[4,5]}$ This method of weight estimation does not take body habitus into account. The formula is: ideal body weight $(\mathrm{kg})=$ height $(\mathrm{cm})-100$.

\section{Buckley method}

Buckley et al.$^{\left[{ }^{[6]}\right.}$ developed formulas to calculate total body weight using $\mathrm{AC}$ and TC measurements of patients. ${ }^{[6]}$ The formulas are gender specific, with estimated male total body weight $=-47.8+(0.78 \times \mathrm{AC})+(1.06 \times$ TC) and estimated female total body weight $=$ $-40.2+(0.47 \times \mathrm{AC})+(1.30 \times \mathrm{TC})$.

\section{Statistical analysis}

The weight estimation methods were compared with the patients' actual weight, thereby estimating the accuracy of each method. Three major outcomes were considered and tested for each weight estimation method and for the body mass index (BMI) subgroups: underweight $\left(<18.5 \mathrm{~kg} / \mathrm{m}^{2}\right)$, normal weight (18.5 - $\left.24.9 \mathrm{~kg} / \mathrm{m}^{2}\right)$, overweight $\left(25-29.9 \mathrm{~kg} / \mathrm{m}^{2}\right)$, obese $\left(30-39.9 \mathrm{~kg} / \mathrm{m}^{2}\right)$ and morbidly obese $\left(\geq 40 \mathrm{~kg} / \mathrm{m}^{2}\right)$. For each of the weight estimation techniques, bias was assessed using mean percentage error (MPE), precision was measured using the limits of agreement (LOA) and root mean square percentage error (RMSPE) and accuracy were measured using the percentage of weight estimations falling within $10 \%$ and $20 \%$ of the patient's actual weight (PW10 and PW20, respectively). ${ }^{[7]}$

\section{Ethical approval}

Approval to conduct this study was obtained from the Human Research Ethics Committee of the University of the Witwatersrand, Johannesburg, SA (ref. no. M160956). Written informed consent was obtained from all patients.

\section{Results}

One hundred and ninety participants were enrolled in the study. Two participants were excluded owing to missing data points. The median (interquartile range (IQR)) age of the population was $36(27 ; 46)$ years. The median (IQR) BMI for the study was $27 \mathrm{~kg} / \mathrm{m}^{2}(23 ; 32)$.

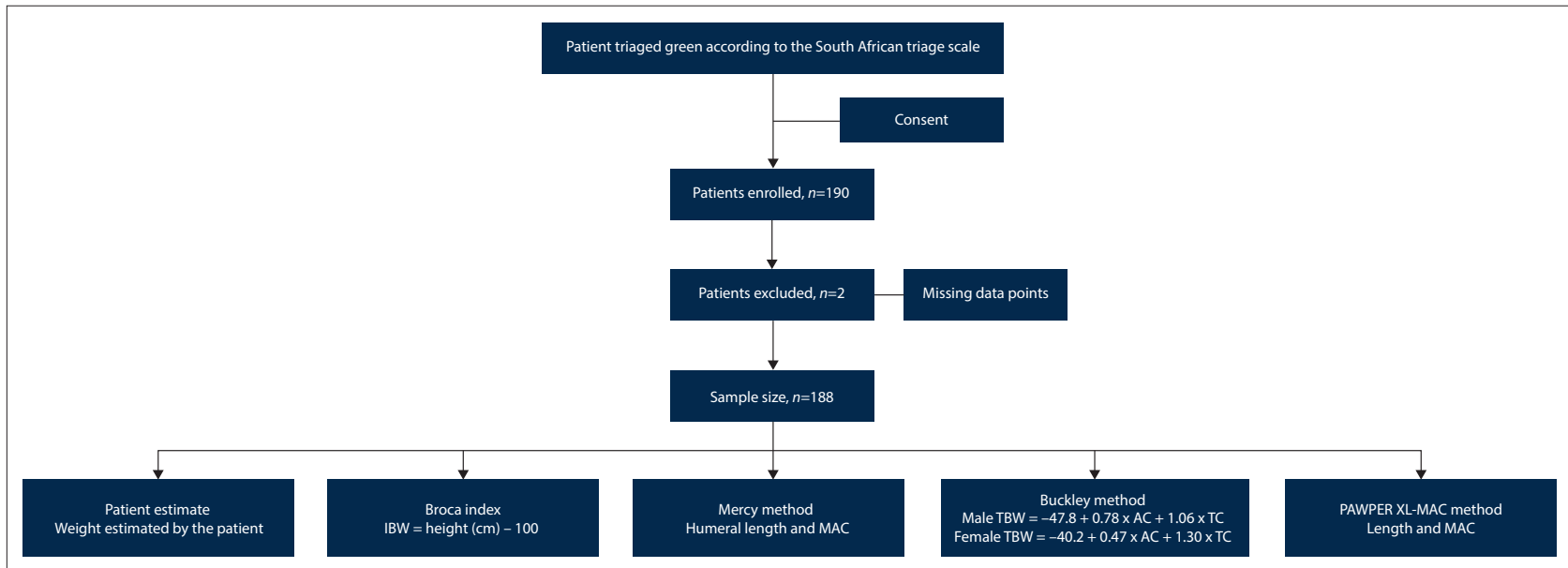

Fig. 1. Patient enrolment and description of variables obtained for weight estimation using each method. $(I B W=$ ideal body weight; humeral length $=$ proximal edge of the acromion to the tip of the olecranon; $M A C=$ mid-arm circumference; $T B W=$ total body weight; $A C=$ abdominal circumference (measured at the umbilicus); $T C=$ thigh circumference $(10 \mathrm{~cm}$ above the superior pole of the patella); PAWPER XL-MAC = paediatric advanced weight prediction in the emergency room eXtra length/eXtra large mid-arm circumference.) 
The demographic and anthropometric data of the study population are shown in Table 1.

Overall bias, precision and accuracy for each of the weight estimation methods are presented in Table 2. Eleven patients were too tall for the PAWPER XL-MAC tape and in 2 patients the humeral length or MAC was greater than the available parameters on the Mercy partial weight table.

Table 3 presents the bias, precision and accuracy analysis of all the weight estimation systems presented according to each BMI category.

\section{Table 1. Demographic and anthropometric distribution of participants}

\begin{tabular}{llll}
\hline & Male, $\boldsymbol{n}(\%)$ & Female, $\boldsymbol{n}(\%)$ & Total, $\boldsymbol{N}(\%)$ \\
\hline BMI $\left(\mathrm{kg} / \mathrm{m}^{2}\right)$ & $65(34.6)$ & $123(65.4)$ & $188(100)$ \\
$<18.5$ & $7(3.7)$ & $3(1.6)$ & $10(5.3)$ \\
$18.5-24.9$ & $24(12.8)$ & $41(21.8)$ & $65(34.6)$ \\
$25.0-29.9$ & $22(11.7)$ & $33(17.5)$ & $55(29.2)$ \\
$30.0-39.9$ & $11(5.9)$ & $29(15.4)$ & $40(21.3)$ \\
$\geq 40.0$ & $1(0.6)$ & $17(9.0)$ & $18(9.6)$
\end{tabular}

Table 2. Summary of the overall bias, precision and accuracy for all the methods of weight estimation

\begin{tabular}{llllll}
\hline & Patient estimate, \% & Broca index, \% & Mercy method, \% & Buckley method, \% & PAWPER XL-MAC method, \% \\
\hline MPE & -4.5 & -0.8 & -1.9 & -15.3 & -3.5 \\
RMSPE & 10 & 19.8 & 10.1 & 16.7 & 11.4 \\
LOA & $-36.0-27.0$ & $-49.3-47.8$ & $-28.7-24.9$ & $-44.2-13.7$ & $-34.0-27.0$ \\
PW10 & 67.6 & 30.3 & 62.2 & 24.5 & 60.1 \\
PW20 & 86.1 & 61.7 & & 72.3 & 85.1 \\
&
\end{tabular}

Table 3. Analysis for all the weight estimation methods for each BMI category, indicating measures of bias, precision and accuracy for each weight estimation technique

\begin{tabular}{|c|c|c|c|c|c|c|c|}
\hline & BMI category & PW 10, \% & PW20, \% & MPE, \% & RMSPE, \% & LLOA, \% & ULOA, \% \\
\hline \multirow[t]{5}{*}{ Patient estimate } & Underweight & 60.0 & 60.0 & 7.7 & 17.8 & -45.2 & 60.5 \\
\hline & Normal weight & 69.5 & 89.8 & -2.6 & 8.2 & -33.8 & 28.5 \\
\hline & Overweight & 83.7 & 89.8 & -4.8 & 7.1 & -25.8 & 16.3 \\
\hline & Obese & 51.4 & 81.1 & -8.8 & 12.4 & -40.0 & 22.6 \\
\hline & Morbidly obese & 55.6 & 88.9 & -8.0 & 11.4 & -44.4 & 78.5 \\
\hline \multirow[t]{5}{*}{ Broca index } & Underweight & 0.0 & 10.0 & 44.9 & 44.9 & 16.7 & 73.1 \\
\hline & Normal weight & 21.5 & 66.2 & 17.9 & 18.7 & -8.1 & 44.0 \\
\hline & Overweight & 72.7 & 94.5 & -2.6 & 8.1 & -22.6 & 17.4 \\
\hline & Obese & 5.0 & 47.5 & -21.9 & 21.9 & -38 & -5.1 \\
\hline & Morbidly obese & 5.6 & 5.6 & -41.1 & 41.1 & -61.9 & 20.4 \\
\hline \multirow[t]{5}{*}{ Mercy method } & Underweight & 60.0 & 80.0 & 16.4 & 18.3 & -35.6 & 68.3 \\
\hline & Normal weight & 78.5 & 98.5 & 0 & 6.9 & -17.6 & 17.7 \\
\hline & Overweight & 52.7 & 90.9 & -1.6 & 11.0 & -28.5 & 25.3 \\
\hline & Obese & 52.5 & 90.0 & -4.9 & 9.8 & -26.6 & 16.9 \\
\hline & Morbidly obese & 44.4 & 72.2 & -13.4 & 14.9 & -35.9 & 9.1 \\
\hline \multirow[t]{5}{*}{ Buckley method } & Underweight & 20.0 & 40.0 & -14.1 & 32.2 & -105.1 & 76.8 \\
\hline & Normal weight & 23.1 & 64.6 & -16.4 & 16.8 & -33.0 & 0.1 \\
\hline & Overweight & 30.9 & 87.3 & -13.1 & 13.4 & -27.7 & 1.5 \\
\hline & Obese & 27.5 & 77.5 & -14.5 & 15.7 & -43.8 & 14.8 \\
\hline & Morbidly obese & 16.7 & 72.2 & -19.9 & 19.9 & -51.6 & 11.8 \\
\hline \multirow[t]{5}{*}{ PAWPER XL-MAC method } & Underweight & 50.0 & 70.0 & 14.8 & 21.7 & -33.5 & 75.2 \\
\hline & Normal weight & 70.8 & 96.9 & 0.9 & 7.4 & -17.9 & 19.6 \\
\hline & Overweight & 70.9 & 94.5 & -0.8 & 8.1 & -20.8 & 19.1 \\
\hline & Obese & 57.5 & 95.0 & -7.8 & 10.7 & -26.7 & 11.0 \\
\hline & Morbidly obese & 0 & 0 & -31.6 & 31.6 & -45.2 & -18.1 \\
\hline
\end{tabular}




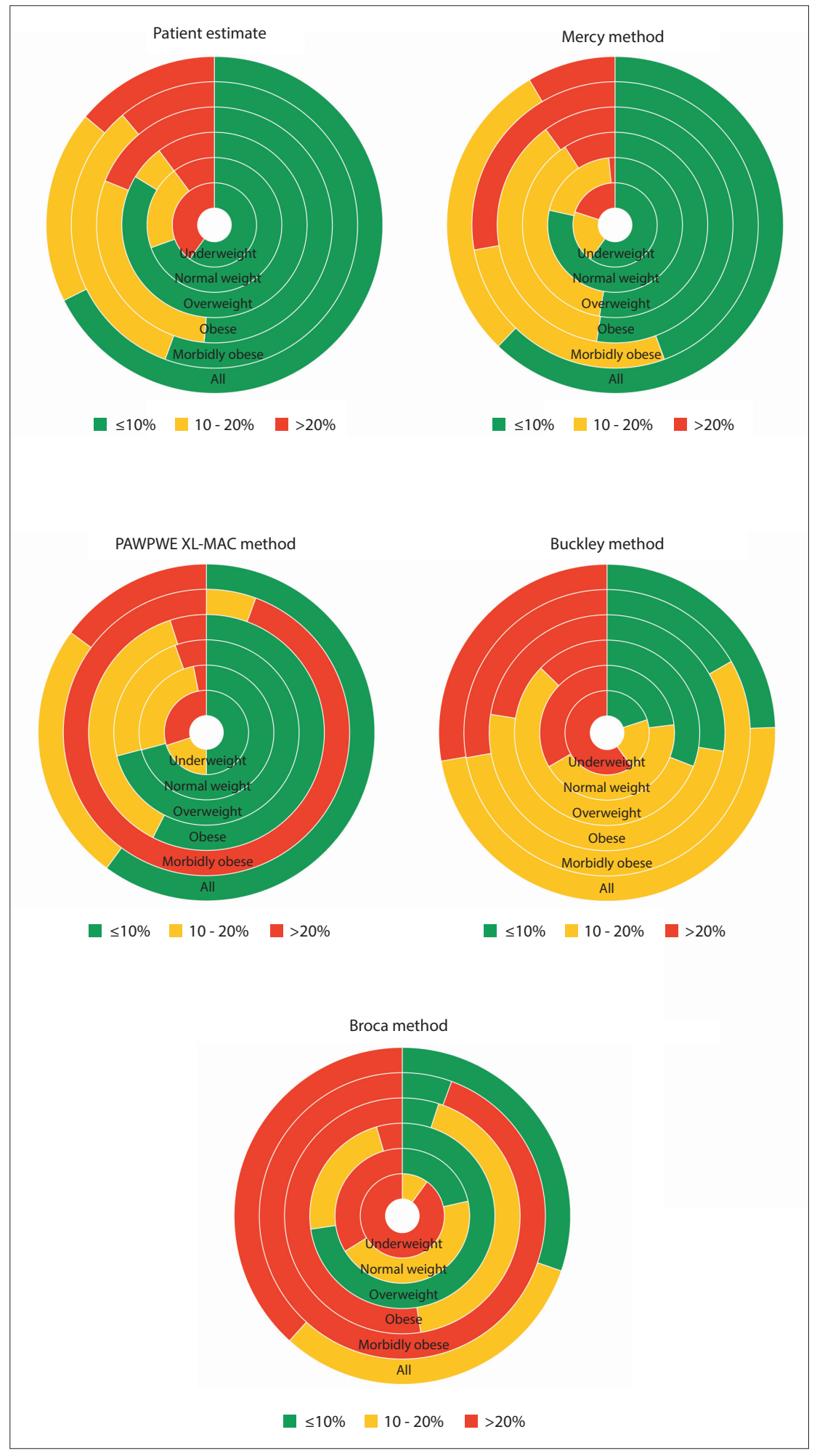

Fig. 2. Radial bar chart, with percentage of weight estimations $<10 \%$ (depicted in green), percentage of weight estimations falling between $10 \%$ and $20 \%$ (depicted in yellow) and critical inaccuracy with percentage of weight estimations $>20 \%$ of actual weight (depicted in red). (PAWPER XL-MAC = paediatric advanced weight prediction in the emergency room eXtra length/eXtra large mid-arm circumference.)

Comparisons from Table 3 are presented in Fig. 2 in a radial bar chart with percentage of weight estimations of $<10 \%$ depicted in green, percentage of weight estimations between $10 \%$ and $20 \%$ depicted in yellow and critical inaccuracy with percentage of weight estimations of $>20 \%$ of actual weight depicted in red. The overall dominance of red or green is a useful way of evaluating the weight estimation methods.

\section{Discussion}

Patient management has evolved from onesize-fits-all to person-centred care. ${ }^{[13]}$ This fundamental concept must also apply to medication dosing in the adult population. To accurately dose medication for adult patients, an accurate weight is required. As a measured weight may not always be available, an alternative method of accurately estimating weight is necessary.

Ideally, the acceptable level of accuracy for a weight estimation system is a PW $10>70 \%$ and a PW20 $>95 \%{ }^{[7]}$ A weight estimation error of $>20 \%$ of the patient's actual weight is considered critical in the paediatric population because of the resultant high risk of harm from medication errors. ${ }^{[14]}$ These standards are also relevant in the adult population, where significant under-dosing of medication may lead to ineffectual drug therapy and overdosing may result in adverse effects, both ultimately resulting in patient morbidity and mortality ${ }^{[15]}$ For example, incorrect dosing of alteplase in a patient with an acute ischaemic stroke is associated with increased adverse events. ${ }^{[16]}$ None of the weight estimation methodologies evaluated in this study achieved a PW10 $>70 \%$. Neither the Mercy nor the PAWPER XL-MAC methods of weight estimation achieved a PW20 >95\% in the overall patient population. In certain BMI categories, however, they both performed very well.

The Broca and Buckley methods both performed dismally and cannot be recommended for general use in the realm of adult weight estimation. The poor performance of formulaic calculations of body weight has previously been demonstrated in paediatric populations. ${ }^{[17]}$ Although the Broca index was originally designed to predict ideal body weight, in this study it did not correlate with a so-called normal BMI. Instead, it performed better in overweight patients. This is contrary to its original calibration, which has been shown to correlate with a BMI of 22.5. ${ }^{[4]}$

Patient weight self-estimation has previously been shown to be more reliable than healthcare provider estimates. ${ }^{[2,18]}$ Patient self-estimates of their weight performed similarly well in this study compared with those in previous studies. ${ }^{[2,18]}$ There was, however, a less than desired level of accuracy, with their estimates falling below the requisite $70 \%$ for PW10. Interestingly, patients in the overweight BMI category performed best in their weight self-estimates, while underweight, obese and morbidly 
obese patients performed the worst. It can potentially be postulated that overweight patients are more aware of their actual weight, as they attempt correcting it towards a healthier BMI.

Under some circumstances, critically ill or injured patients may not be able to provide an estimation of their own weight - the need to estimate weight is unavoidable. It is therefore important to identify the best possible methods for this purpose. In this study the dual length- and habitus-based paediatric weight estimation methods performed reasonably well, although not as well as was desirable.

Both the Mercy and PAWPER XL-MAC methods of weight estimation use the MAC as a surrogate for body habitus determination to accurately account for body composition and habitus differences among patients. These systems, however, were both designed for use in children. Body composition of children is different from that of adults. ${ }^{[19]}$ For these paediatric methodologies to perform equally well in the adult and paediatric populations, the differences would need to be accounted for by a recalibration of the measurements, adjusting for adult body composition. ${ }^{[20]}$ From a technical point of view, the PAWPER XL-MAC methodology might be easier to recalibrate than the Mercy method. This is because the PAWPER XL-MAC method performed well in normal weight and overweight patients, but less well at extremes of habitus. The potential recalibration would require a separate study and subsequent validation. This may lead to estimates in these categories that could potentially increase the accuracy of this method in adults. We speculate that the accuracy of the PAWPER system could be improved to achieve a PW $10>75 \%$ and a PW20 $>95 \%$. Since the Mercy method has a far less precise association with body composition than the PAWPER XL-MAC method, the recalibration process would most likely be far more complex in the former. ${ }^{[20]}$ The recalibration would also need to account for taller adults (i.e. $>180 \mathrm{~cm}$ ) and the larger humeral lengths and/or MAC in adults on the Mercy partial weight table. The potential recalibration for these weight estimation systems would need to be done in a properly designed derivation/validation study with independent samples.

\section{Study limitations}

One of the limitations of this study was that it was performed in relatively healthy adults. The effects of underlying acute or chronic illness on weight estimation accuracy are unknown and will need to be established in future studies. Furthermore, the changes in body composition associated with increasing age were not addressed, but will be a very interesting avenue for future studies.

\section{Conclusions}

None of the evaluated weight estimation methodologies was accurate enough for use in adult weight estimation. The Mercy and PAWPER XL-MAC methods both showed significant promise, but need further refinement. Although patient self-estimates were similarly accurate to those found in previous studies, they were not an accurate option; these estimations would remain the first choice if the patient was able to provide one. The Broca index and Buckley method cannot be recommended due to their poor performance.
Declaration. The research for this study was done in partial fulfilment of the requirements for OA's MMed (Emergency Medicine) degree at the University of the Witwatersrand.

Acknowledgements. None.

Author contributions. LG, MW and OA conceived and designed the study. OA collected the data. All authors conducted the analysis. OA drafted the original manuscript. LG and MW contributed to the interpretation of the data and composition of the final manuscript. All authors reviewed and approved the final version of the manuscript.

Funding. None.

Conflicts of interest. Prof. Mike Wells is the inventor of the PAWPER XLMAC tape; however, these tapes are sold on a not-for-profit basis to cover the costs of production.

1. Darnis S, Fareau N, Corallo CE, Poole S, Dooley MJ, Cheng AC. Estimation of body weight in hospitalised patients. QJM 2012;105(8):769-774. https://doi.org/10.1093/qjmed/hcs060

2. Hall WL 2nd, Larkin GL, Trujillo MJ, Hinds JL, Delaney KA. Errors in weight estimation in the emergency department: Comparing performance by providers and patients. J Emerg Med 2004;27(3):219-224. https://doi.org/10.1016/j.jemermed.2004.04.008

3. Kahn CA, Oman JA, Rudkin SE, Anderson CL, Sultani D. Can ED staff accurately estimate the weight of adult patients? Am J Emerg Med 2007;25(3):307-312. https://doi.org/10.1016/..ajem.2006.08.010

4. Weber-Sanchez A, Ortega Sofia V, Weber-Alvarez P. Validation of the Broca index as the most practical Weber-Sanchez A, Ortega Sofia V, Weber-Alvarez P. Validation of the Broca index as the most practical
method to calculate the ideal body weight. J Clin Invest Stud 2018;1(1):1-4. https:// 1 oi.org/10.15761/ method to calc

5. Laurent I, Astère M, Paul B, et al. The use of Broca index to assess cut-off points for overweight in adults: A short review. Rev Endocr Metab Disord 2020;21:521-526. https://doi.org/10.1007/s11154020-09566-5

6. Buckley RG, Stehman CR, Dos Santos FL, et al. Bedside method to estimate actual body weight in the emergency department. J Emerg Med 2012;42(1):100-104. https://doi.org/10.1016/j. jemermed.2010.10.022

7. Wells M, Goldstein LN, Bentley A. The accuracy of emergency weight estimation systems in children - a systematic review and meta-analysis. Int J Emerg Med 2017;10(1):29. https://doi.org/10.1186/s12245systematic re $017-0156-5$

8. Wells M, Goldstein LN, Bentley A. Development and validation of a method to estimate body weight Wells M, Goldstein $\mathrm{LN}$, Bentley A. Development and validation of a method to estimate body weight
in critically ill children using length and mid-arm circumference measurements: The PAWPER XLMAC system. S Afr Med J 2017;107(11):1015-1021. https://doi.org/10.7196/SAMJ.2017.v107i11.12505 9. Abdel-Rahman SM, Ridge AL. An improved pediatric weight estimation strategy. Open Med Dev J 2012;4:87-97. https://doi.org/10.2174/1875181401204010087

10. Cattermole GN, Manirafasha A. Estimating weight in patients of all-ages: A prospective study in Rwanda. medRxiv. https://doi.org/10.1101/2020.02.29.20029371

11. Abdel-Rahman SM, Ahlers N, Holmes A, et al. Validation of an improved pediatric weight estimation strategy. J Pediatr Pharmacol Ther 2013;18(2):112-121. https://doi.org/10.5863/1551-6776-18.2.112

12. Wells M. A validation of the PAWPER XL-MAC tape for total body weight estimation in preschool children from low- and middle-income countries. PLoS ONE 2019;14(1):e0210332. https://doi.
ches org/10.1371/journal.pone.0210332

13. Kaehne A. Care integration - from 'one size fits all' to person centred care comment on 'achieving integrated care for older people: shuffling the deckchairs or making the system watertight for the future?' Int J Health Policy Manag 2018;7(10):955-957. https://doi.org/10.15171/ijhpm.2018.51

14. Wells M, Goldstein L, Bentley A. A validation study of the PAWPER XL tape: Accurate estimation of both total and ideal body weight in children up to 16 years of age. Trauma Emerg Care 2017;2(4):1-8. https://doi.org/10.15761/TEC.1000141

15. Barras M, Legg A. Drug dosing in obese adults. Aust Prescr 2017;40(5):189-193. https://doi. org/10.18773/austprescr.2017.053

16. Paliwal PR, Sharma AK, Komal Kumar RN, et al. Effect of erroneous body-weight estimation on outcome of thrombolyzed stroke patients. J Thromb Thrombolysis 2020;50:921-928. https://doi. outcome of thrombolyzed stroke
org/10.1007/s11239-020-02118-3

17. Wells $\mathrm{M}$, Goldstein L, Bentley A. It is time to abandon age-based emergency weight estimation in children! A failed validation of 20 different age-based formulas. Resuscitation 2017;116(7):73-83. https://doi.org/10.1016/j.resuscitation.2017.05.018

18. Lin BW, Yoshida D, Quinn J, Strehlow M. A better way to estimate adult patients' weights. Am J Emerg Med 2009;27(9):1060-1064. https://doi.org/10.1016/j.ajem.2008.08.018

19. Kyle UG, Earthman CP, Pichard C, Coss-Bu JA. Body composition during growth in children: Limitations and perspectives of bioelectrical impedance analysis. Eur J Clin Nutr 2015;69(12):1298-1305. https:// doi.org/10.1038/ejcn.2015.86

20. Wells M, Goldstein LN. How and why paediatric weight estimation systems fail - a body composition study. Cureus 2020;12(3):e7198. https://doi.org/10.7759/cureus.7198 